\title{
BMJ Open Association of microalbuminuria and high-normal 24-hour urinary albumin excretion with metabolic syndrome and its components in the general Chinese population: cross-sectional study
}

Jianwei Xu, ${ }^{1}$ Jixiang Ma, ${ }^{2}$ Xiaorong Chen, ${ }^{1}$ Liuxia Yan, ${ }^{1}$ Xiaoning Cai, ${ }^{1}$ Xiaolei Guo, ${ }^{3}$ Yongqing Zhang, ${ }^{4}$ Jing $\mathrm{Wu}^{1}$

To cite: $X u$ J, Ma J, Chen $X$ et al. Association of microalbuminuria and highnormal 24-hour urinary albumin excretion with metabolic syndrome and its components in the general Chinese population: cross-sectional study. BMJ Open 2019;9:e031443. doi:10.1136/ bmjopen-2019-031443

- Prepublication history for this paper is available online. To view these files, please visit the journal online (http://dx.doi org/10.1136/bmjopen-2019031443).

Received 04 May 2019 Revised 30 September 2019 Accepted 11 October 2019

Check for updates

(c) Author(s) (or their employer(s)) 2019. Re-use permitted under CC BY-NC. No commercial re-use. See rights and permissions. Published by BMJ.

For numbered affiliations see end of article.

Correspondence to

Dr Jing Wu;

wujing@ncncd.chinacdc.cn

\section{ABSTRACT}

Objective Microalbuminuria (MAU) has been described as a risk factor for metabolic syndrome (MetS). However, the association between MetS components with MAU and 24hour urinary albumin excretion (UAE) has not been clearly explained in the general Chinese population. We aimed to analyse the associations between MAU and high-normal 24-hour UAE with MetS and its components.

Design Cross-sectional observational study.

Setting Four selected counties/districts in China's Shandong and Jiangsu Provinces.

Participants A total of 2261 participants aged 18-69 years were included in this study. Participants with missing physical examination data or incomplete urine collection were not included in the analysis.

Results The prevalence of MAU was $9 \%$, and the mean 24-hour UAE was $18 \mathrm{mg} / \mathrm{d}$. The prevalence of MAU was significantly higher for the MetS, high blood pressure (BP), high triglyceride (TG) levels, low high-density lipoprotein cholesterol (HDL-C) and hyperglycaemia groups but not for the central obesity group. Both MAU and mean 24-hour UAE were significantly increased in association with a number of MetS components. The adjusted prevalence OR (POR) for MetS with MAU was 2.95 (95\% Cl 2.15 to 4.04) compared with those without MAU. MAU was significantly associated with three components of MetS: high BP (POR=1.86, 95\% Cl 1.31 to 2.64), high TG levels ( $P O R=1.80,95 \% \mathrm{Cl} 1.31$ to 2.46) and hyperglycaemia ( $\mathrm{POR}=1.84,95 \% \mathrm{Cl} 1.34$ to 2.53 ). No significant association between MAU and central obesity or low HDL-C was found. The presence of MetS gradually increased according to the normal-range 24hour UAE quartiles: $\mathrm{POR}=1.00, \mathrm{POR}=1.22$, $\mathrm{POR}=1.14$ and $\mathrm{POR}=2.02$, respectively. Hyperglycaemia also increased significantly according to the normal-range 24-hour UAE quartiles.

Conclusions MAU and elevated 24-hour UAE within the normal range were closely associated with MetS in the Chinese population, which may provide a basis for the development of early interventions to decrease the effects of MetS.

\section{Strengths and limitations of this study}

- We used 24-hour urinary albumin excretion (UAE) to define microalbuminuria (MAU), which is more accurate than most previous studies.

- This is the largest sample size of the general Chinese population to collected 24-hour urine.

- We explored the association between high-normal 24-hour UAE with MetS and its components.

- A causal relationship between MAU and MetS cannot be demonstrated in our cross-sectional study.

\section{INTRODUCTION}

Microalbuminuria (MAU), defined by an abnormally high albumin excretion $(30-300 \mathrm{mg} / \mathrm{d})$ in a 24-hour urine sample, was significantly associated with chronic kidney disease, cardiovascular disease and progression of end-stage renal disease independent of traditional risk factors. ${ }^{1-4}$ Prospective and epidemiological studies have found that MAU is also a powerful predictor of all-cause and cardiovascular mortality in the general population. ${ }^{56}$

Metabolic syndrome (MetS) is a widely accepted description of a cluster of metabolic abnormalities characterised by obesity, hypertension, dyslipidaemia and hyperglycaemia. ${ }^{7}$ Some studies have evaluated the relationship between MetS and MAU as a marker for earlystage chronic kidney disease. ${ }^{8-11}$ Significant associations between MetS and MAU have been demonstrated in Japanese, ${ }^{9}$ Korean ${ }^{10} 12$ and Chinese populations. ${ }^{13-16}$ However, data concerning the relationship between individual MetS components and MAU have been inconsistent, and a causal relationship between MAU and MetS remains unclear despite the predictive value MAU showed in the aforementioned studies. Furthermore, 
studies on the association between normal-range 24-hour urinary albumin excretion (UAE) and MetS components have been limited. ${ }^{15}$

Twenty-four-hour UAE level is considered the 'gold standard' for defining MAU. ${ }^{17}{ }^{18}$ However, most of the previous studies of MAU in the Chinese population have used an early morning or random spot urine sample instead of measuring 24-hour UAE. Therefore, in this study, we investigated the prevalence of MAU by analysing 24-hour UAE and analysed the association of MAU and normal-range 24-hour UAE with MetS and its components.

\section{METHODS}

\section{Study participants}

Data were derived from the supplemental baseline survey of the Shandong Ministry of Health Action on Salt Reduction and Hypertension project, which was a cross-sectional survey conducted at four sites in the Shandong and Jiangsu provinces during 2013 and 2014. We used a stratified, multistage sampling method to select the participants. We selected 80 villages or communities from four sites using proportional probability sampling. A random sample of 120 adults aged 18-69 years was drawn from each village or community. A total of 9600 people were selected, and 8995 of these individuals participated in the survey (response rate of $93.7 \%$ ). A total of 605 replacements were selected from all individuals in the same village or community after excluding the already selected participants. A total of 9600 individuals participated in the survey and physical examination. A random sample of at least 30 adults was drawn among 120 adults from each village or community. A total of 2480 people were selected, and 2295 participated in the survey (response rate of $92.5 \%$ ). Of the 185 non-responders, 113 were replaced by adults with similar profiles from the same community or village. Finally, a single 24-hour urine sample was collected from a subsample of 2408 participants. Participants with the following conditions were not required to provide urine samples: (1) patients who had difficulty collecting a urine sample; (2) patients with an acute or chronic urinary infection; (3) women who were pregnant, breastfeeding or actively menstruating; and (4) patients with severe vomiting or diarrhoea. We excluded three subjects with missing data from their physical examination or blood samples and 127 participants with incomplete 24-hour urine collection. For the purpose of the present study on MAU, we also excluded 17 subjects with macroalbuminuria or a 24-hour UAE of $>300 \mathrm{mg} / \mathrm{d}$. Therefore, a total of 2261 participants were included in this study.

Written informed consent was obtained from all participants.

\section{Demographic, anthropometrical and biochemical data collection}

A face-to-face interview was conducted by local trained health professionals using a standard questionnaire. Relevant variables included age, sex, educational level, smoking status, alcohol intake, regular exercise, and previous diagnosis and treatment of hypertension and diabetes. During the physical examination, height, weight, waist circumference (WC) and blood pressure (BP) were measured by trained researchers using standardised protocols and techniques. Weight and height were measured with participants dressed in light, indoor clothing without shoes using standardised techniques and calibrated equipment. WC was measured at the narrowest point between the lower border of the rib cage and the iliac crest. The body mass index (BMI) was calculated as the weight in kilogram divided by the height in metre squared $\left(\mathrm{kg} / \mathrm{m}^{2}\right)$. BP was measured three times by an electronic sphygmomanometer (HEM-7071, Omron Corporation, Japan), and the final BP was obtained by averaging the three measurements.

Fasting blood samples collected from each participant were processed and shipped in cold storage to a certified laboratory (ADICON Clinical Laboratory, Jinan, China). Fasting blood glucose (FBG), total cholesterol (TC), triglyceride (TG), high-density lipoprotein cholesterol (HDL-C) and low-density lipoprotein cholesterol were measured. Plasma glucose was measured using a modified hexokinase enzymatic method. Serum cholesterol and TG levels were analysed enzymatically using commercially available reagents.

\section{4-Hour urine collection and analysis}

Eligible participants were instructed not to change their dietary or lifestyle habits. We provided a standard plastic container for each participant to collect a 24-hour urine sample. Trained researchers gave each participant both written and verbal instructions on how to collect a 24-hour urine sample. Health professionals carefully explained to the subjects the purpose of the 24-hour urine collection and asked the subjects to correctly repeat the information. The exact 24-hour urine collection time, including starting and ending times, was recorded by the supervising health professional. The total volume of the collection was measured by a laboratory technician, and urine aliquots were frozen at $-20^{\circ} \mathrm{C}$ for approximately 30 days and were shipped to the ADICON Clinical Laboratory in Jinan. Urinary creatinine was measured by the picric acid method. UAE was measured with an immunonephelometric method using the Olympus AU640 Analyser, for which the coefficient of variation was $3 \%$. Either a 24-hours urinary volume less than $500 \mathrm{~mL}$ or a 24-hour urinary creatinine volume that was $\pm 2 \mathrm{SD}$ outside of the sex-specific mean, $0.98-16.17 \mathrm{mmol} / \mathrm{L}$ for men and $0.93-$ $13.60 \mathrm{mmol} / \mathrm{L}$ for women, was defined as an incomplete urine collection. ${ }^{19}$

\section{Definition of MetS}

We adopted the harmonised criteria of MetS, which defines MetS as the presence of $\geq 3$ of the following risk factors $^{20}$ : central obesity defined as a WC of $\geq 90 \mathrm{~cm}$ in men and $\geq 80 \mathrm{~cm}$ in women; high $\mathrm{BP}$ defined as a systolic blood pressure (SBP) of $\geq 130 \mathrm{~mm} \mathrm{Hg}$, a diastolic blood 
pressure (DBP) of $\geq 85 \mathrm{~mm} \mathrm{Hg}$ or treatment with an antihypertensive medication; high TG levels defined as a fasting plasma TC level of $\geq 1.7 \mathrm{mmol} / \mathrm{L}$ or drug treatment for increased TC; low HDL-C defined as HDL-C of $<1.0 \mathrm{mmol} / \mathrm{L}$ in men and $<1.3 \mathrm{mmol} / \mathrm{L}$ in women or drug treatment for low HDL-C; and hyperglycaemia defined as an FBG level of $\geq 5.6 \mathrm{mmol} / \mathrm{L}$ or drug treatment for increased FBG.

\section{Statistical analysis}

Continuous variables are presented as the mean (SD), and categorical variables are presented as percentages. According to their quartiles of 24-hour UAE in a normal range $(n=2058)$, study subjects were divided into four groups: Q1 $(0-9.38 \mathrm{mg} / \mathrm{d})$, Q2 $(9.39-11.96 \mathrm{mg} / \mathrm{d})$, Q3 (11.97-15.46 mg/d) and Q4 (15.47-29.99 mg/d).

We performed logistic regression analyses to study the association between MAU and 24-hour UAE with MetS and its components while controlling for covariates, including sociodemographic factors (age, sex and education level) and lifestyle factors (regular exercise, alcohol intake and smoking). Participants who did not have MAU or who were in the Q1 group were used as a reference group to estimate the prevalence ORs (PORs) and 95\% CIs. Tests of linear trends across increasing quartiles of 24-hour UAE were conducted by treating the medians of the average 24-hour $\mathrm{UAE}$ as a continuous variable in the logistic regression models. Statistical analyses were performed with SAS V.9.3. The tests performed were two-sided, and a $p$ value of $<0.05$ was considered statistically significant.

\section{Patient and public involvement}

Patients and the public were not involved in the design or planning of the study.

\section{RESULTS}

\section{Characteristics of subjects}

Among the 2261 participants, the prevalence of MAU was $9 \%$ (203), and the prevalence was not significantly different between men and women $(8.8 \%$ vs $9.1 \%$, $\mathrm{p}=0.08$ ). The mean 24-hour UAE was $18.0 \mathrm{mg} / \mathrm{d}$. The population characteristics are summarised according to MAU and normal-range 24-hour UAE quartiles in table 1.

Table 1 General characteristics of the study participants

\begin{tabular}{|c|c|c|c|c|c|c|c|c|}
\hline & \multicolumn{4}{|c|}{ Normal range 24-hour UAE (mg/d) } & \multirow[b]{2}{*}{$P$ value } & \multicolumn{2}{|c|}{ Microalbuminuria } & \multirow[b]{2}{*}{$P$ value } \\
\hline & Q1 & Q2 & Q3 & Q4 & & No & Yes & \\
\hline Number of subjects & 515 & 515 & 516 & 512 & & 2058 & 203 & \\
\hline Age (years) & $41.7(13.5)$ & $42.5(13.5)$ & $41.8(13.5)$ & $42.5(13.3)$ & 0.67 & $42.1(13.5)$ & $41.4(13.5)$ & 0.48 \\
\hline Men $(\%)$ & 51.8 & 48.2 & 50.8 & 48.4 & 0.57 & 49.8 & 48.8 & 0.78 \\
\hline $\begin{array}{l}\text { Body mass index }(\mathrm{kg} / \\
\left.\mathrm{cm}^{2}\right)\end{array}$ & $24.4(3.6)$ & $24.6(3.6)$ & $24.7(3.9)$ & $25.5(3.9)$ & $<0.001$ & $24.8(3.8)$ & $26.0(4.5)$ & $<0.001$ \\
\hline $\begin{array}{l}\text { Waist circumference } \\
\text { (cm) }\end{array}$ & $82.3(9.1)$ & $82.4(9.6)$ & $82.7(9.9)$ & $84.8(10.5)$ & $<0.001$ & $83.0(9.8)$ & $85.8(12.5)$ & $<0.001$ \\
\hline $\begin{array}{l}\text { Systolic blood } \\
\text { pressure }(\mathrm{mm} \mathrm{Hg})\end{array}$ & $129.5(19.4)$ & $130.7(18.5)$ & $129.8(18.7)$ & $133.1(20.7)$ & 0.0111 & $130.8(19.4)$ & $136.1(23.3)$ & $<0.001$ \\
\hline $\begin{array}{l}\text { Diastolic blood } \\
\text { pressure }(\mathrm{mm} \mathrm{Hg})\end{array}$ & $81.8(11.4)$ & $83.1(10.8)$ & $82.5(11.4)$ & 84.9 (12.2) & $<0.001$ & $83.1(11.5)$ & $87.9(14.4)$ & $<0.001$ \\
\hline High-school (\%) & 24.1 & 21.9 & 23.1 & 25.4 & 0.61 & 23.6 & 26.6 & 0.34 \\
\hline Smoking (\%) & 30.1 & 29.1 & 29.1 & 27.3 & 0.80 & 28.9 & 33.0 & 0.22 \\
\hline Alcohol (\%) & 23.7 & 27.6 & 26.2 & 27.3 & 0.47 & 26.2 & 27.1 & 0.78 \\
\hline Regular exercise (\%) & 17.3 & 16.7 & 22.3 & 22.3 & 0.03 & 19.6 & 30.5 & $<0.001$ \\
\hline $\begin{array}{l}\text { Fasting blood glucose } \\
(\mathrm{mmol} / \mathrm{L})\end{array}$ & $5.6(1.1)$ & $5.6(1.0)$ & $5.7(1.1)$ & $6.0(1.7)$ & $<0.001$ & $5.7(1.3)$ & $6.5(2.4)$ & $<0.001$ \\
\hline $\begin{array}{l}\text { Total cholesterol } \\
(\mathrm{mmol} / \mathrm{L})\end{array}$ & $4.7(0.9)$ & $4.8(0.9)$ & $4.8(0.9)$ & $4.9(1.0)$ & 0.0281 & $4.8(0.9)$ & $5.1(1.0)$ & $<0.001$ \\
\hline $\begin{array}{l}\text { High-density } \\
\text { lipoprotein (mmol/L) }\end{array}$ & $1.3(0.3)$ & $1.3(0.3)$ & $1.3(0.3)$ & $1.2(0.3)$ & 0.0203 & $1.3(0.3)$ & $1.2(0.3)$ & 0.03 \\
\hline $\begin{array}{l}\text { Low-density } \\
\text { lipoprotein (mmol/L) }\end{array}$ & $2.4(0.7)$ & $2.4(0.6)$ & $2.5(0.7)$ & $2.5(0.7)$ & 0.0186 & $2.4(0.7)$ & $2.5(0.7)$ & 0.06 \\
\hline Triglyceride (mmol/L) & $1.4(1.2)$ & $1.4(1.3)$ & $1.5(1.4)$ & $1.8(2.1)$ & $<0.001$ & $1.6(1.6)$ & $2.3(2.4)$ & $<0.001$ \\
\hline Creatinine $(\mathrm{mmol} / \mathrm{d})$ & $6.0(2.2)$ & $7.4(2.6)$ & $8.0(3.2)$ & $8.6(3.1)$ & $<0.001$ & $7.5(3.0)$ & $8.8(3.1)$ & $<0.001$ \\
\hline 24-hour UAE (mg/d) & $7.7(1.2)$ & $10.7(0.8)$ & $13.5(1.0)$ & $20.1(3.7)$ & $<0.001$ & $13.0(5.0)$ & $68.8(50.4)$ & $<0.001$ \\
\hline
\end{tabular}

UAE, urinary albumin excretion. 


\begin{tabular}{|c|c|c|c|}
\hline \multirow[b]{2}{*}{ Components } & \multicolumn{2}{|l|}{ MAU (\%) } & \multirow[b]{2}{*}{$P$ value } \\
\hline & No $(n=2058)$ & Yes $(n=203)$ & \\
\hline \multicolumn{4}{|l|}{ Central obesity } \\
\hline No & 91.49 & 8.51 & 0.1982 \\
\hline Yes & 89.76 & 10.24 & \\
\hline \multicolumn{4}{|c|}{ High blood pressure } \\
\hline No & 93.52 & 6.48 & $<0.001$ \\
\hline Yes & 88.85 & 11.15 & \\
\hline \multicolumn{4}{|c|}{ High triglycerides } \\
\hline No & 93.11 & 6.89 & $<0.001$ \\
\hline Yes & 86.18 & 13.82 & \\
\hline \multicolumn{4}{|c|}{ Low high-density lipoprotein cholesterol } \\
\hline No & 91.81 & 8.19 & 0.0249 \\
\hline Yes & 88.72 & 11.28 & \\
\hline \multicolumn{4}{|c|}{ Hyperglycaemia } \\
\hline No & 93.94 & 6.06 & $<0.0001$ \\
\hline Yes & 87.78 & 12.22 & \\
\hline \multicolumn{4}{|l|}{ MetS } \\
\hline No & 93.52 & 6.48 & $<0.0001$ \\
\hline Yes & 84.89 & 15.11 & \\
\hline
\end{tabular}

MAU, microalbuminuria; MetS, metabolic syndrome.

Compared with those without MAU, participants with MAU were more likely to have higher WC, BMI, SBP, DBP, FBG and TG. Similarly, these variables were also significantly different among increasing quartiles of 24-hour UAE.

\section{MAU and 24-hour UAE by the number of MetS components}

The prevalence of MAU in individuals with MetS and its components is shown in table 2. The prevalence of MAU was significantly higher in the MetS, high-BP, high-TG levels and hyperglycaemia groups. The prevalence rates of MAU among subjects with $0(n=342)$, one $(n=632)$, two $(\mathrm{n}=632)$, three $(\mathrm{n}=433)$, and four or five $(\mathrm{n}=222)$ components of MetS were $5.0 \%, 5.5 \%, 8.2 \%, 14.5 \%$ and $16.2 \%$, respectively. The corresponding mean 24-hour UAE measurements were 14.5, 15.1, 17.9, 22.7 and $22.8 \mathrm{mg} / \mathrm{d}$, respectively. Both the prevalence rate of MAU and the mean 24-hour UAE were significantly elevated according to the number of MetS components with all $\mathrm{p}$ values being $<0.001$ (figure 1 ).

\section{Association between MAU and MetS components}

The associations between MAU and the components of MetS are shown in table 3. Compared with participants without MAU, the age-adjusted and sex-adjusted POR for MetS with MAU was 2.93 (95\% CI 2.15 to 4.00), and the multivariate-adjusted POR was 2.95 (95\% CI 2.15 to 4.04). For MetS components, in both the age-adjusted and sex-adjusted and multivariate-adjusted models, MAU was strongly associated with high BP, high TG levels and
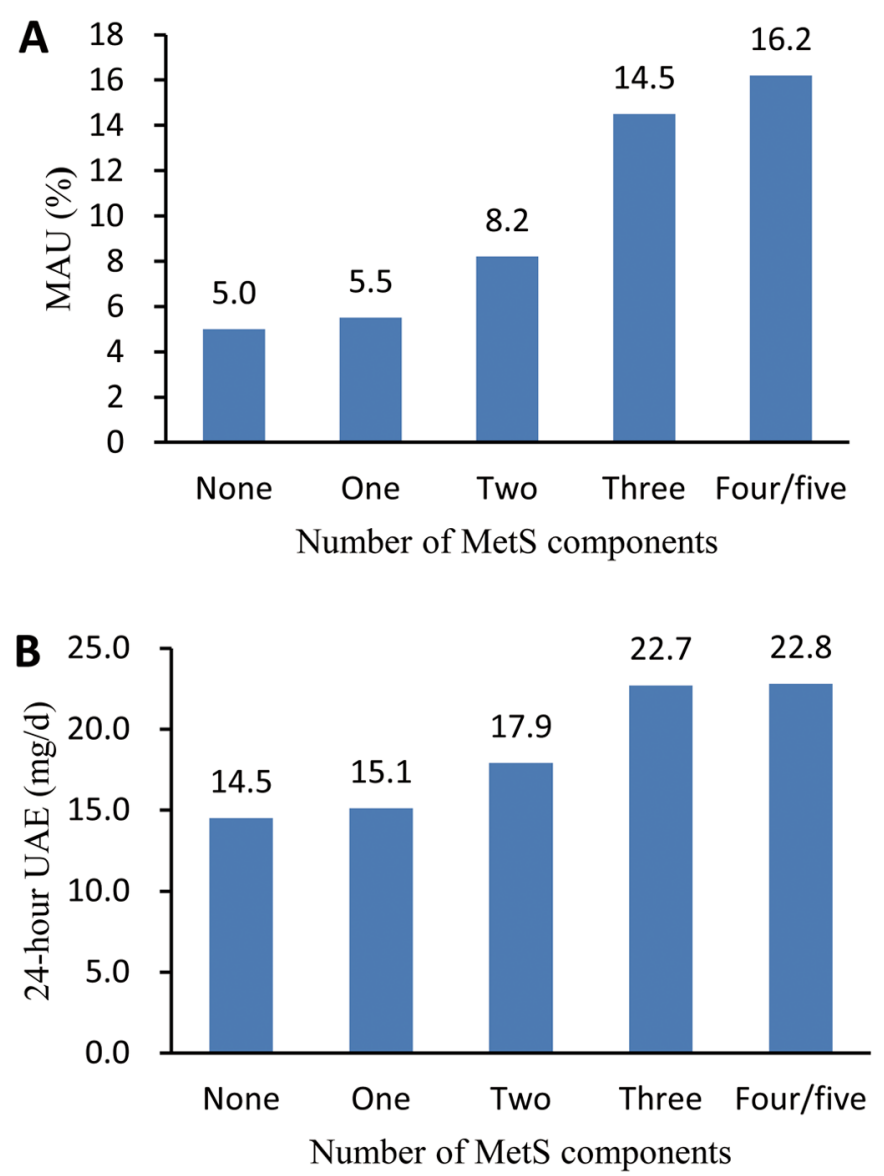

Figure 1 Prevalence of MAU (A) and mean 24-hour UAE (B) according to the number of MetS components. MAU, microalbuminuria; MetS, metabolic syndrome; UAE, urinary albumin excretion.

hyperglycaemia. However, no significant association between MAU and central obesity or low HDL-C was found.

\section{Associations between high-normal 24-hour UAE and MetS}

Table 4 shows that the odds of MetS gradually increased according to the normal-range 24-hour UAE quartiles. The multivariate-adjusted PORs of MetS were 1.22, 1.14 and 2.02 for 24-hour UAE quartiles 2, 3 and 4, respectively, compared with the lowest quartile $(p<0.0001)$. Furthermore, compared with the lowest 24-hour UAE quartile, the multivariate-adjusted POR of the highest quartile was 1.52 for hyperglycaemia $(\mathrm{p}<0.01)$. However, no significant associations between normal-range 24-hour $\mathrm{UAE}$ and the other components of MetS were found.

\section{DISCUSSION}

Our study showed that the prevalence of MAU was $9.0 \%$ in the study population and was much more prevalent in persons with MetS (15.1\%) in particular. The prevalence of MAU in our study was moderate compared with that in other studies in China $(4.1 \%-15 \%) \cdot{ }^{13}{ }^{1421-24}$ This varied prevalence observed in the Chinese population may be 
Table 3 Relationship between MAU and MetS components

\begin{tabular}{lccll} 
& & & MAU & \\
\cline { 4 - 5 } & Cases (n) & Without MAU & Model 1* & Model 2† \\
\hline MetS & 655 & 1.00 & $2.93(2.15$ to 4.00$)$ & $2.95(2.15$ to 4.04$)$ \\
Central obesity & 615 & 1.00 & $1.40(0.92$ to 2.13$)$ & $1.02(0.65$ to 1.60$)$ \\
High blood pressure & 1211 & 1.00 & $2.20(1.57$ to 3.09$)$ & $1.86(1.31$ to 2.64$)$ \\
High triglycerides & 680 & 1.00 & $2.21(1.65$ to 2.97$)$ & $1.80(1.31$ to 2.46$)$ \\
$\begin{array}{l}\text { Low high-density lipoprotein } \\
\text { cholesterol }\end{array}$ & 576 & 1.00 & $1.71(1.13$ to 2.60$)$ & $1.32(0.85$ to 2.04$)$ \\
Hyperglycaemia & 1072 & 1.00 & $2.28(1.68$ to 3.11$)$ & $1.84(1.34$ to 2.53$)$ \\
\hline
\end{tabular}

${ }^{*}$ Model 1: adjusted for age and sex.

†Model 2: adjusted for age, sex, education attainment, regular exercise, alcohol consumption, smoking and for the other components of MetS (except for analyses of MetS).

MAU, microalbuminuria; MetS, metabolic syndrome.

associated with different age distributions, regions and methods of defining MAU.

In the current study, the prevalence of MAU was consistently higher in persons with MetS than in those without MetS. The prevalence of MAU increased significantly with increasing numbers of MetS components after the subjects were divided by the number of MetS components. The same results were obtained in previous studies. ${ }^{12-15} 2526$ This finding indicates that a large number of participants with MAU are easily overlooked in routine health examinations that do not include MAU measurements. This finding also suggests that intervention for MetS should be initiated at the earliest stage of renal injury.

Furthermore, our study showed that MAU was significantly associated with MetS and its components, apart from central obesity and low HDL-C. Many epidemiological studies have suggested an independent association between MAU and MetS. However, findings on the

Table 4 Normal-range 24 hours' urinary albumin excretion quartiles associated with MetS and its components

\begin{tabular}{|c|c|c|c|c|c|}
\hline & \multicolumn{4}{|c|}{ Prevalence ORs $(95 \% \mathrm{Cl})$} & \multirow[b]{2}{*}{$P$ value } \\
\hline & Q1 & Q2 & Q3 & Q4 & \\
\hline \multicolumn{6}{|l|}{ MetS } \\
\hline Model $2 \dagger$ & 1.00 & $1.22(0.90$ to 1.65$)$ & 1.14 (0.84 to 1.55$)$ & 2.02 (1.51 to 2.72 ) & $<0.0001$ \\
\hline \multicolumn{6}{|c|}{ Central obesity } \\
\hline Model 2† & 1.00 & 1.29 (0.89 to 1.88$)$ & 1.07 (0.73 to 1.58$)$ & 1.53 (1.04 to 2.25$)$ & 0.1221 \\
\hline \multicolumn{6}{|c|}{ High blood pressure } \\
\hline Model $1^{*}$ & 1.00 & 1.18 (0.90 to 1.54$)$ & 0.99 (0.75 to 1.29$)$ & 1.43 (1.09 to 1.87$)$ & 0.0253 \\
\hline Model 2† & 1.00 & 1.12 (0.85 to 1.48$)$ & 0.93 (0.71 to 1.23$)$ & 1.24 (0.94 to 1.64$)$ & 0.1957 \\
\hline \multicolumn{6}{|c|}{ High triglycerides } \\
\hline Model $1^{*}$ & 1.00 & 1.37 (0.96 to 1.93$)$ & 1.28 (0.90 to 1.81$)$ & 1.55 (1.09 to 2.20$)$ & 0.0953 \\
\hline Model 2† & 1.00 & 1.34 (0.93 to 1.94$)$ & 1.26 (0.87 to 1.83$)$ & 1.29 (0.89 to 1.87$)$ & 0.4045 \\
\hline \multicolumn{6}{|c|}{ Hyperglycaemia } \\
\hline Model $1^{*}$ & 1.00 & $1.12(0.87$ to 1.44$)$ & 1.37 (1.07 to 1.77$)$ & 1.72 (1.33 to 2.21$)$ & 0.0001 \\
\hline Model $2 \dagger$ & 1.00 & $1.09(0.84$ to 1.41$)$ & 1.34 (1.03 to 1.74$)$ & $1.52(1.17$ to 1.98$)$ & 0.0055 \\
\hline
\end{tabular}

*Model 1: adjusted for age and sex.

†Model 2: adjusted for age, sex, education attainment, regular exercise, alcohol consumption, smoking and for the other components of MetS (except for the analyses of MetS).

MetS, metabolic syndrome. 
associations between various components of MetS and MAU are controversial. BP and fasting glucose were consistently found to be two main risk factors associated with MAU, which was also clearly shown in our study. However, the associations between MAU and abdominal obesity, HDL and TG levels were inconsistent in previous studies. ${ }^{8916}$ Thus far, two studies have reported an association between MAU and risk of MetS, with ORs of 5.13 (95\% CI 1.96 to 13.45 ) and 2.71 (95\% CI 1.69 to 4.36$),{ }^{14}$ which is consistent with our finding.

Our study further showed that the prevalence of MetS gradually increased with increasing normal-range 24-hour UAE quartiles, which is consistent with previous studies. ${ }^{1526-28}$ Thus far, only one prospective cohort study reported HRs with 95\% CIs for MetS: 1.57 (95\% CI 1.14 to 2.18) for the three highest albumin:creatinine ratio quartiles compared with the lowest one. ${ }^{28}$ However, the association between the components of MetS and highnormal 24-hour UAE were not exactly the same. Ge et al reported that the relationship between 24-hour UAE within the normal range and central obesity, elevated BP and elevated TG levels was significant. ${ }^{15}$ Another study also found that the association between low-grade albuminuria and the components of MetS, except for low HDL-C, was significant. ${ }^{25}$ In our study, a relationship between high-normal 24-hour UAE and hyperglycaemia in the general Chinese population was found. This association persisted after adjustment for multiple risk factors.

In our study, we found that not only MAU but also elevated 24-hour UAE within the normal range had a significant relationship with an increased risk of MetS in Chinese adults. The magnitude of this association persisted after controlling for traditional risk factors. Our finding has important public health implications for preventing MetS. ${ }^{15}$ The prevalence of MetS in China was $24.2 \%^{29}$ and is becoming a serious public health problem in China. Our findings demonstrated that reducing 24-hour UAE even within the normal range should be an important priority for reducing the effects of MetS. Our study suggested that clinicians should carefully evaluate the risk of MetS in individuals with normal-range UAE. Annual MAU screening in the MetS population should be an essential part of our preventive medicine efforts. Policy makers should support such screening as part of the universal coverage policy programmes.

Our study had strengths. First, we used 24-hour UAE to define MAU in a relatively large sample population, which was more accurate than most previous studies in the general Chinese population. Furthermore, this population-based epidemiological study found an association between high-normal 24-hour UAE and MetS, which is different from most previous studies in China. In addition, our study rigorously used standardised methods and quality control for data collection. However, our study had several limitations. First, due to investigative convenience, we did not use an objective biomarker, such as para-aminobenzoic acid, to assess complete 24-hour urine. $^{30}$ We assessed the completeness by measuring urinary volume and creatinine concentration in the present study. Second, we did not evaluate the renal function of the subjects, which may have caused some bias in the results. Third, although multiple covariates were included in the adjustment, some potential confounding factors, such as dietary intake ${ }^{31}$ and other medications, could not be ruled out. Finally, because this study had a cross-sectional design, it was difficult to interpret a causal association between MAU and 24-hour UAE with MetS and its components. Therefore, future prospective studies are recommended to confirm our findings.

\section{CONCLUSIONS}

Our study confirmed that both MAU and high-normal 24-hour UAE were strong risk factors for MetS in the general Chinese population. The assessment of these risk factors can result in an opportunity for early intervention to decrease the effects of MetS in China.

\section{Author affiliations}

${ }^{1}$ National Center for Chronic and Noncommunicable Disease Control and Prevention, Chinese Center for Disease Control and Prevention, Beijing, China

${ }^{2}$ Office of NCD and Ageing Health Management, Chinese Center for Disease Control and Prevention, Beijing, China

${ }^{3}$ Department of Non-communicable Disease Control and Prevention, Shandong Center for Disease Control and Prevention, Jinan, China

${ }^{4}$ Department of Non-communicable Disease Control and Prevention, Jiangsu Center for Disease Control and Prevention, Nanjing, China

Acknowledgements Special thanks to Professor Jixiang Ma for his great contribution to the project initiation. We would like to express our sincere appreciation to all staff for performing the field work. We are also grateful to all the study participants.

Contributors JM, XCh and JW designed the study and supervised the data collection. JX, LY, XCa, XG and YZ participated in field work and data collection. $J X$ and $L Y$ analysed the data. JX wrote the manuscript to which all the authors contributed. JM and JW critically revised the manuscript for important intellectual content. All the authors reviewed and approved the final manuscript.

Funding The survey was supported by Shandong-Ministry of Health Action on Salt Reduction and Hypertension (number 2013) and the Young Scholar Scientific Research Foundation of the Chinese Center for Disease Control and Prevention (number 2018A203).

Competing interests None declared.

Patient consent for publication Not required.

Ethics approval The survey was approved by the ethics committee of the National Center for Chronic and Noncommunicable Disease Control and Prevention, Chinese Center for Disease Control and Prevention (201311).

Provenance and peer review Not commissioned; externally peer reviewed. Data availability statement Data are available upon reasonable request.

Open access This is an open access article distributed in accordance with the Creative Commons Attribution Non Commercial (CC BY-NC 4.0) license, which permits others to distribute, remix, adapt, build upon this work non-commercially, and license their derivative works on different terms, provided the original work is properly cited, appropriate credit is given, any changes made indicated, and the use is non-commercial. See: http://creativecommons.org/licenses/by-nc/4.0/.

\section{REFERENCES}

1 Matsushita K, van der Velde M, Astor BC, et al. Association of estimated glomerular filtration rate and albuminuria with all-cause and cardiovascular mortality in general population cohorts: a collaborative meta-analysis. Lancet 2010;375:2073-81. 
2 Gansevoort RT, Matsushita K, van der Velde M, et al. Lower estimated GFR and higher albuminuria are associated with adverse kidney outcomes. A collaborative meta-analysis of general and highrisk population cohorts. Kidney Int 2011:80:93-104.

3 Astor BC, Matsushita K, Gansevoort RT, et al. Lower estimated glomerular filtration rate and higher albuminuria are associated with mortality and end-stage renal disease. A collaborative meta-analysis of kidney disease population cohorts. Kidney Int 2011;79:1331-40.

4 van der Velde M, Matsushita K, Coresh J, et al. Lower estimated glomerular filtration rate and higher albuminuria are associated with all-cause and cardiovascular mortality. A collaborative meta-analysis of high-risk population cohorts. Kidney Int 2011;79:1341-52.

5 Chen Y-Y, Li Y-Y, Lu Y-H, et al. Albuminuria independently predicts cardiovascular and all-cause mortality in a middle-aged and elderly Chinese population. Scand J Clin Lab Invest 2012;72:281-6.

6 Yuyun MF, Khaw K-T, Luben R, et al. Microalbuminuria independently predicts all-cause and cardiovascular mortality in a British population: the European prospective investigation into cancer in Norfolk (EPIC-Norfolk) population study. Int J Epidemiol 2004;33:189-98.

7 Alberti KGMM, Zimmet P, Shaw J. Metabolic syndrome--a new world-wide definition. A Consensus Statement from the International Diabetes Federation. Diabet Med 2006;23:469-80.

8 Palaniappan L, Carnethon M, Fortmann SP. Association between microalbuminuria and the metabolic syndrome: NHANES III. Am J Hypertens 2003;16:952-8.

9 Hao Z, Konta T, Takasaki S, et al. The association between microalbuminuria and metabolic syndrome in the general population in Japan: the Takahata study. Intern Med 2007;46:341-6.

10 Choi HS, Ryu SH, Lee K-B. The relationship of microalbuminuria with metabolic syndrome. Nephron Clin Pract 2006;104:c85-93.

11 Klausen KP, Parving $\mathrm{H}-\mathrm{H}$, Scharling $\mathrm{H}$, et al. The association between metabolic syndrome, microalbuminuria and impaired renal function in the general population: impact on cardiovascular disease and mortality. J Intern Med 2007;262:470-8.

12 Lee H-O, Bak H-J, Shin J-Y, et al. Association between metabolic syndrome and microalbuminuria in Korean adults. Korean $J$ Fam Med 2015;36:60-71.

13 Chen B, Yang D, Chen Y, et al. The prevalence of microalbuminuria and its relationships with the components of metabolic syndrome in the general population of China. Clin Chim Acta 2010;411:705-9.

14 Sheng C-S, Hu B-C, Fan W-X, et al. Microalbuminuria in relation to the metabolic syndrome and its components in a Chinese population. Diabetol Metab Syndr 2011;3.

$15 \mathrm{Ge} \mathrm{Z}$, Guo X, Chen X, et al. Are microalbuminuria and elevated $24 \mathrm{H}$ urinary Microalbumin excretion within normal range associated with metabolic syndrome in Chinese adults? PLoS One 2015;10:e0138410.

16 Lee Y-Y, Yang C-K, Weng Y-M, et al. All components of metabolic syndrome are associated with microalbuminuria in a Chinese population. PLoS One 2016;11:e0157303.
17 Thoenes M, Bramlage P, Khan BV, et al. Albuminuria: pathophysiology, epidemiology and clinical relevance of an emerging marker for cardiovascular disease. Future Cardiol 2007;3:519-24.

18 Crews DC, Boulware LE, Gansevoort RT, et al. Albuminuria: is it time to screen the general population? Adv Chronic Kidney Dis 2011;18:249-57.

$19 \mathrm{Xu} \mathrm{J}$, Chen X, Ge Z, et al. Associations of usual 24-hour sodium and potassium intakes with blood pressure and risk of hypertension among adults in China's Shandong and Jiangsu provinces. Kidney Blood Press Res 2017:42:188-200.

20 Alberti KGMM, Eckel RH, Grundy SM, et al. Harmonizing the metabolic syndrome: a joint interim statement of the International diabetes Federation Task force on epidemiology and prevention; National heart, lung, and blood Institute; American heart association; world heart Federation; international atherosclerosis Society; and international association for the study of obesity. Circulation 2009;120:1640-5.

21 Shan Y, Zhang Q, Liu Z, et al. Prevalence and risk factors associated with chronic kidney disease in adults over 40 years: a population study from central China. Nephrology 2010;15:354-61.

22 Hao G, Wang Z, Zhang L, et al. Prevalence of microalbuminuria among middle-aged population of China: a multiple center cardiovascular epidemiological study. Angiology 2015;66:49-56.

23 Chen W, Chen W, Wang H, et al. Prevalence and risk factors associated with chronic kidney disease in an adult population from southern China. Nephrol Dial Transplant 2009;24:1205-12.

24 Yan L, Ma J, Guo X, et al. Urinary albumin excretion and prevalence of microalbuminuria in a general Chinese population: a crosssectional study. BMC Nephrol 2014;15:165.

25 Zhang J, Chen Y, Xu Y, et al. Low-Grade albuminuria is associated with metabolic syndrome and its components in middle-aged and elderly Chinese population. PLoS One 2013;8:e65597.

26 Li M-F, Feng Q-M, Li L-X, MF L, LX L, et al. High-Normal urinary albumin-to-creatinine ratio is independently associated with metabolic syndrome in Chinese patients with type 2 diabetes mellitus: a cross-sectional community-based study. J Diabetes Investig 2015;6:354-9.

27 Park S-Y, Park Y-K, Cho K-H, et al. Normal range albuminuria and metabolic syndrome in South Korea: the 2011-2012 Korean National health and nutrition examination survey. PLoS One 2015;10:e0125615.

28 Oh C-M, Park SK, Kim HS, et al. High-Normal albuminuria predicts metabolic syndrome in middle-aged Korean men: a prospective cohort study. Maturitas 2014;77:149-54.

29 Li Y, Zhao L, Yu D, et al. Metabolic syndrome prevalence and its risk factors among adults in China: a nationally representative crosssectional study. PLoS One 2018;13:e0199293.

30 Murakami K, Sasaki S, Takahashi Y, et al. Sensitivity and specificity of published strategies using urinary creatinine to identify incomplete 24-h urine collection. Nutrition 2008;24:16-22.

31 Julibert A, Bibiloni MDM, Tur JA. Dietary fat intake and metabolic syndrome in adults: a systematic review. Nutr Metab Cardiovasc Dis 2019;29:887-905. 